\title{
Standesangelegenheiten.
}

\section{Heranziehung der Aerzte zur Gewerbesteuer?}

\author{
Von J. Schwalbe.
}

Die schwierige Finanzlage Hessens treibt seine gesetzgebenden Körperschaften seit einiger Zeit, nach neuen Steuerzuflüssen zu suchen. Als eine ergiebige Einnahmequelle glauben einige Mitglieder des hessischen Finanzausschusses der Zweiten Kammer die Belastung der Rechts. anwälte und Aerzte mit der Gewerbesteuer betrachten zu dürfen. In dem von der Regierung vorgelegten Gesetzentwurf über die Gemeindeumlage sind nach Artikel 8 unter den der Gewerbesteuer nicht unterliegenden Berufen auch die Praxis der Aerzte und der Rechtsanwälte aufgeführt, in derselben Weise wie in dem bisherigen, seit dem Jahre 1884 bestehenden Gewerbesteuergesetz. Diese Ausnahme soll jetzt nach dem Wunsch einiger Abgeordneter ${ }^{1}$ ) aufgehoben werden, und $\mathrm{zwar}$ wird zur Unterstützung des Antrags eine lebhafte Agitation im Lande betrieben.

Derselbe Antrag ist schon bei der ersten Beratung des Gesetzentwurfs im Jahre 1905 gestellt worden; er wurde aber von der Regierung bekämpft und von der Majorität der Zweiten Kammer abgelehnt. Nach der Auffassung der hessischen Aerzte ist es zweifelhaft, ob sich auch diesmal im Finanzausschuß und in den Kammern eine Majorität zugunsten des bisherigen Modus finden wird. Und als eine Unterstützung dieses Bedenkens muß die Tatsache angesehen werden, daß nicht nur die Aerzte, sondern auch die Rechtsanwälte Hessens gegen die drohende Steuer mobil gemacht haben: pflegen doch die Juristen eine feine Witterung für alle gesetzgeberischen Aktionen zu besitzen.

Unter diesem Gesichtswinkel scheint mir eine Betrachtung der Gründe, die gegen die Heranziehung der Aerzte zur Gewerbesteuer sprechen, notwendig zu sein. Uns bewegt dabei nicht bloß der Wunsch, den hessischen Kollegen zu Hilfe zu kommen. Denn wenn es dem hessischen Landtag gelingen sollte, die Steuer der Aerzte und Leidensgenossen durchzudrücken und die Regierung für die Sanktionierung ihres Beschlusses zu gewinnen (noch selten hat der Fiskus eine ihm von der Volksvertretung präsentierte Steuer abgelehnt!), so liegt die Gefahr nicht allzufern, daß die anderen Bundesstaaten diesem edlen Beispiel folgen könnten. Es gilt also hier das Wort: Tua res agitur, und nicht nur die hessischen Kollegen haben Grund, der Steuerschraube ihrer Abgeordneten entgegenzuarbeiten.

Auch im preu Bischen Abgeordnetenhause hat man bei den Beratungen des Entwurfs eines Gewerbesteuergesetzes im Jahre 1890 ähnliche Geliiste gezeigt, wie sie jetzt in Hessen hervortreten. Nach dem Bericht der zur Vorberatung des Gesetzentwurfs eingesetzten Kommission des Abgeordnetenhauses machten die Gegner der Gewerbesteuerfreiheit der Aerzte (und Rechtsanwälte) geltend den gewinnreichen, oft geradezu glänzenden Geschäftsbetrieb dieser Berufsstände, der, verglichen mit der Notlage der kleineren Gewerbetreibenden, das im Gesetzentwurfe verliehene Privilegium ebenso unnötig wie gehässig erscheinen lasse. Die Aerzte und Rechtsanwälte seien früher in Preußen als Gewerbetreibende besteuert worden, diese Besteuerung finde heute noch in mehreren Ländern statt, der Arzt werde in der Gewerbeordnung für das Deutsche Reich ausdrücklich genannt. Wenn man für die fraglichen Berufe als Grund der Steuerbefreiung ihre ideale Richtung aufrufe, so sei diese für jedes ehrliche Gewerbe, welches in seiner Weise der Volkswohlfahrt diene, in Anspruch zu nehmen, übrigens sei weder in dem Berufe der Rechtsanwälte noch demjenigen der Aerzte das Ideale das Ausschließliche oder auch nur Vorwiegende, das Geschäfte machen und Geld verdienen werde auch hier oft zur Hauptsache.

Von den Vertretern der Staatsregiemng wurde hierauf entgegnet: Die ältere Steuergesetzgebung habe allerdings auch den Beruf des Rechtsanwaltes und Arztes erfaßt, man habe aber unter dem Drucke der öffentlichen Meinung diese Besteuerung in dem Gewerbesteuergesetze von 1820 wieder fallen lassen müssen. Der Arzt wie der Rechtsanwalt arbeite in Ausübung einer Wissenschaft für ein ideales Ziel; wie der Rechtsanwalt das

1) nicht, wie es irrtümlich in dem "Brief aus Hessen", No. 1, S. 36 hies, nach dem Antrag der Regierung. 
Recht zu sichern, so sei der Arzt die Krankheiten zu heilen berufen. Daß für die Betätigung dieses Berufes ein Gewinn erzielt, Geld verdient werde, sei notwendig, aber nicht die Hauptsache; hierdurch dürfe der Beruf des Rechtsanwaltes und Arztes nicht zu einem bloß auf den Ertrag gestellten Gewerbe herabsinken. Wo der gewerbliche Charakter in der Rechtshilfe oder Krankenbehandlung nnzweideutig sei, wie bei den Rechtskonsulenten und sogenannten Naturärzten, unterlägen die betreffenden Geschäftsbetriebe der Gewerbesteuer. Uebrigens werde auch der Arzt für Nebengeschäfte, welche nicht in seinen Beruf notwendig hineinfielen, bereits besteuert, z. B. beim Selbstdispensieren von Arzneimitteln, beim Betriebe von mit Pension verbundenen Privatkliniken etc.

Von Mitgliedern der Kommission wurde zugunsten der Steuerbefreiung der Rechtsanwälte und Aerzte noch bemerkt, daß deren im öffentlichen Interesse zu forderndes Ansehen dadurch in einzelnen Fällen gesunken sei, daß sie in vorwiegender Erwerbsucht ihren Beruf des idealen Charakters entkleidet haben; der sichere Weg, um diese bedauerliche Erscheinung, wenn auch nur scheinbar zu rechtfertigen, würde eingeschlagen werden, wollte man die Rechtsanwaltschaft und die ärztliche Praxis durch Unterwerfung unter die Gewerbesteuer als einen ihrem Wesen nach auf den Gewerbsgewinn gerichteten Beruf kennzeichnen.

Bei den Verhandlungen über den Gesetzentwurf im Plenum des Abgeordnetenhauses trat unser unvergeßlicher Kollege Graf mit Entschiedenheit für die Befreiung der Aerzte von der Gewerbestener ein. Er wies mit Nachdruck darauf hin, da B der ärztliche Beruf nicht mit dem Maßstab eines Gewerbebetriebs zu messen sei. ,Der Arzt soll - das ist seine heilige Pflicht _- das Interesse des Patienten höher stellen als sein eigenes Interesse. Denn mit welchem Recht könnte sonst ein Patient, wenn er das nicht voraussetzte, dem Arzte seine höchsten Güiter, Leben und Gesundheit, anvertrauen! Der Arzt soll keine Reklame machen, keine geschäftlichen Anpreisungen erlessen, - das ist bei uns in Deutschland, Gott sei Dank, noch die Regel für die große Mehrzahl der deutschen Aerzte. Und vor allem, meine Herren, erinnere ich Sie an die Tätigkeit des Arztes auf dem Gebiete der öffentlichen Gesundheitspflege; hier soll er den Krankheiten vorbeugen, die Epidemien verhüten oder sie im Keime ersticken. Durch diese in den meisten Fällen unentgeltlich ausgeübte Tätigkeit schädigt der Arzt aber direkt seine eigene Erwerbsquelle. Die vorliegende Frage ist deshalb nicht etwa eine Frage des materiellen Interesses, sie ist auch nicht nur eine Frage der Vornehmheit; - denn der Stand allein macht noch nicht vornehm, sondern erst die Art, wie man ihn vertritt; - es handelt sich bei dieser Steuerbefreiung darum, daß Sie damit anerkenn:n, der Aorztestand habe gewisse Berufspllichten, welche dem allgemeinen Wohle dienen.'

Wirksamer und überzeugender noch als die Beweisführung vom e thischen Standpunkte sind die Schlüsse, die aus der Stellung der Gesetzgebung und der Rechtsprechung für die Frage zu ziehen sind, ob der ärztliche Beruf überhaupt ein Gewerbe darstellt.

Schon in den Motiven zum Entwurf des preuBischen Gewerbesteuergesetzes heißt es zu $\S 4$ No. 7, ,daß die von der Steuer zu befreienden Erwerbszweige, u. a. namentlich der ärztliche Beruf, nach der die preußische Gesetzgebung von jeher beherrschenden Auffassung sowie nach der allgemeinen Volksanschauung nicht zu den Gewerben gehören und hier lediglich aufgeführt worden sind, um etwaigen Mißdeutungen vorzubeugen".

In völligem Einklang mit dieser Auffassung der preußischen Regierung hat das Reichsgericht gerade in den letzten Jahren zu wiederholten Malen sich dahin entschieden, daß der ärztliche Beruf außerhalb der Gewerbeordnung stehe. Von diesen Urteilen führen wir das vom 11. Juni 1907 in seinen wesentlichsten Teilen wörtlich an, um der auch noch in ärztlichen Kreisen immer wieder auftauchenden Ansicht, daß die ärztliche Tätigkeit nach dem Gesetze als ein Gewerbe angesehen sei, weil sie in $\S 29$ der Gewerbeordnung aufgeführt werde, ein fïr allemal den Boden zu entziehen. Das Reichsgericht sagt: ,Die Ausübung der Heilkunde auf Grund staatlicher Approbation ist, wenngleich sie meist im Wege des privat. rechtlichen Vertrages geleistet und honoriert wird, ihrem inneren und eigentlichen Wesen nach (abgesehen von gewerblich betriebenen Privatkrankenanstalten) kein gewerbliches Unternehmen. Zunächst nicht im Sinne der Gewerbeordnung. Denn die Gewerbeordnung hat einen bestimmten Begriff des Gewerbes nicht aufgestellt und nicht aufstellen wollen. Es ist also der in der steuerrechtlichen Entsch. d. RG. in Zivils. Bd. 39 S. 138 als völlig entscheidend bezeichnete Umstand, daß die Abs. 3 und 5 des $\$ 29$ Gew.-O. vom "Gewerbebetrieb" der approbierten Aerzte sprechen, für die Frage nach der inneren Natur des ärztlichen Berufes ohne Belang. Die Eimbeziehung der Medizinalpersonen in die Gewerbeordnung hette nach den Motiven zu $\$ 29$ den Zweck, denselben die Freizïgigkeit innerhalb des Bundesgebietes zu ermöglichen und zu gewährleisten; deshalb sind die Prüfungen und Approbationen der Medizinalpersonen von Reichs wegen geordnet. Satz 2 des Abs. 1 des $\S 6$ Gew.-O. besagt demgemäß, da $B$ das Gesetz auf die Ausübung der Heilkunde nur insoweit Anwendung findet, als dasselbe ausdrückliche Bestimmungen darüber enthält. Aus der Gewerbeordnung ist hiernach für die Natur des ärztlichen Berufes positiv nichts zu gewinnen. Eine positive Bekundung über das Wesen des ärztlichen Berufes ist dagegen gegeben in den Standesanschaunngen, welche in den den praktischen Aerzten staatlich erteilten Organisationen unter staatlicher Autorität durchgeführt werden. Der für Preußen auf Grund des Gesetzes betreffend die ärztlichen Ehrengerichte, das Umlagerecht und die Kassen der Aerztekammern vom 25. November 1899 seit dem 1. April 1900 bestehende ärztliche Ehrengerichtshof hat in einer Reihe von Entscheidungen Reklamen, Angriffe auf den Besitzstand eines Berufsgenossen, Verbindungen mit Kurpfuschern als standesunwürdig geahndet... Alle diese Sprüche laufen entsprechenden Entscheidungen des Ehrengerichtshofes für deutsche Rechtsanwälte völlig parallel. Beide Ehrengerichtshöfe finden einen Verstoß gegen die Standesehre auch in dem Kauf und Verkauf der Praxis, weil hierdurch der ideelle Wert des persönlichen Vertrauens in Geld umgesetzt werde. Beide Ehrengerichts. höfe rügen also Maßnahmen, welche erlaubte und gewöhnliche Geschäfte eines gewerblichen Unternehmens sind, beim Arzt und Rechtsanwalt als Berufspflichtverletzung... Die sich unter staatlicher Autorität durchsetzende sittliche Ueberzeugung und die tatsächliche Sitte der deutschen Aerzte gehen dahin, da B der ärztliche Beruf, wie der Rechtsanwaltsberuf, als "Gewerbe", d. h. als eine Einnahmequelle, als ein auf Geldverdienen gerichtetes Unternehmen nicht ausgeübt wird und nicht ausgeübt werden darf. Die Ueberzeugung von dem höheren sittlichen Zweck beider Berufe greift über die Standeskreise hinaus; sie ist eine allgemeine. Sie tritt hervor in Entscheidungen des Preußischen Oberverwaltungsgerichts und des Kammergerichts. Sie kommt zum Ausdruck in den Motiven zu $\$ 2$ des neuen Handelsgesetzbuchs: ,Der Ausdruck gewerbliches Unternehmen braucht im Gesetz nicht näher erläutert zu werden; schon vermöge der Bedeutung, welche ihm nach dem allgemeinen Sprachgebrauche zukommt, genügt er, um die Ausübung der Kunst, der Rechtsanwaltschaft, des ärztlichen Berufs etc. auszuschließen." Nach den Sittenanschauungen nicht nur der Aerzte und Rechtsanwälte selbst, und nicht nur der sonst höher gebildeten Volkskreise, sondern des gesamten deutschen Volkes stehen die allgemeinen Interessen dienenden Berufe des Arztes und des Rechtsanwalts über dem Niveau einer Gelderwerbstätigkeit und dürfen auf die Stufe eines gewerblichen Unternehmens nicht herabgezogen werden

Das eigentümliche und entscheidende Gepräge beider Berufe liegt darin, daB sie fundamentale, allgemeine, öffentliche Zwecke, nämlich die der Gesundheitspflege und der Rechtspflege, auf Grund staatsseitig geforderter und gewährleisteter wissenschaftlicher Vorbildung unter besonderer Verantwortung zu erfüllen haben. So bemerken die Motive zum I. Entwurf der Gewerbeordnung: Durch Verzicht auf den ärztlichen Befähigungsnachweis würde die Gesetzgebung in tiefen Widerspruch treten mit den berechtigten Anforderungen, welche an die Staatsgewalt im Interesse der Sorge für Leben und Gesundheit der Staatsangehörigen gestellt werden... Mit Recht bemerkt die Entscheidung des ärztlichen Ehrengerichtshofs Ministerialblatt 1903 S. 219: ,Die zu starke Betonung und Hervorhebung des gewerblichen Moments ist geeignet, den Stand in den Augen der Bevölkerung herabzusetzen. Es ist eine Tatsache, daß das sittliche Bewußtsein aller Volkskreise gegen eine Ausnutzung des ärztlichen und anwaltlichen Berufs wie einer Gelderwerbsquelle feinfühlig und lebhaft reagiert als gegen einen Mißbrauch einer öffentlichen, mit Pflichten verbundenen Stellung. Insbesondere ist auch die Stellung des nicht beamteten Arztes durch eine Reihe öffentlichrechtlicher Rechte und Pflichten gekennzeichnet. . . .“

Mit Bezug auf den $\$ 29$ der Gewerbeordnung äuBert sich das Reichsgericht in einem anderen Urteil vom Juni 1907 noch dahin, daß, ,der Senat durch die Gewerbeordnung die Ausübung der ärztlichen Berufstätigkeit nur nach ihrer gewerbepolizeilichen Seite hin für geregelt ansieht, ohne daß dadurch die auf wissenschaftlicher Grundlage beruhende Berufstätigkeit des approbierten Arztes selbst zum Gewerbe gemacht ist“"

Im Einklang damit heißt es in einem weiteren Gerichtsurteil, da B der Arzt, da er kein Gewerbe betreibt, den reichs- und landesgesetzlichen Bestimmungen über die Sonntagsruhe im Gewerbe nicht unterstellt ist.

Ferner ist nach der zitierten Entscheidung des Reichsgerichts vom Juni 1907 die Ausübung der ärztlichen Praxis allein kein die Versicherungspflicht des Personals des Arztes bedingender Gewerbebetrieb.

Und in gleicher Weise urteilt das Reichs-Versicherungsam t (Amtliche Nachrichten 1907, S. 429): ,Aerzte sind nicht als Gewerbetreibende im Sinne des $\$ 14$ Absatz 1, Ziffer 2 des Invaliden-Versicherungsgesetzes anzusehen; deshalb haben die Aerzto nicht das Recht, sich auf Grund des Invaliden-Versicherungsgesetzes freiwillig zu versichern."

Die obersten Instanzen unserer ordentlichen und außerordentlichen Gerichte sind demnach übereinstimmend der Ansicht, da B der ärztliche Beruf kein Gewerbe ist: also darf er auch nicht der Gewerbesteuer unterworfen werden, weder in Hessen, noch in einem andern deutschen Bundesstaat. Von Rechts wegen. 